\title{
Anti-MuSK Positive Myasthenia Gravis with Anti-Lrp4 and Anti-titin Antibodies
}

\author{
Rika Yamashita ${ }^{1}$, Mikito Shimizu ${ }^{1}$, Kousuke Baba ${ }^{1}$, Goichi Beck ${ }^{1}$, Makoto Kinoshita ${ }^{1}$, \\ Tatsusada Okuno ${ }^{1}$, Osamu Higuchi ${ }^{2}$ and Hideki Mochizuki ${ }^{1}$
}

\begin{abstract}
:
In addition to muscle nicotinic acetylcholine receptor (AChR) and muscle-specific kinase (MuSK), lowdensity lipoprotein receptor (Lrp4) has recently been discovered to be a novel target antigen among patients with seronegative myasthenia gravis (MG). We herein report the findings of a 62-year-old patient who showed positivity for anti-MuSK, anti-Lrp4, and anti-titin antibodies. The patient developed MG crisis following a 10-year history of intermittent double vision with ptosis, and a 7-year history of dropped head. Our detailed clinical, laboratory, and therapeutic descriptions highlight its unique characteristics of anti-MuSKantibody positive MG accompanied by anti-Lrp4 and anti-titin antibodies.
\end{abstract}

Key words: myasthenia gravis, anti-MuSK antibody, anti-Lrp4 antibody, anti-titin antibody, steroid pulse therapy

(Intern Med 60: 137-140, 2021)

(DOI: 10.2169/internalmedicine.4957-20)

\section{Introduction}

Myasthenia gravis (MG) is an autoimmune neurological disorder caused by antibodies directed against the neuromuscular junction. Anti-muscle nicotinic acetylcholine receptor (AChR) antibodies (Abs) are known to be the most common Abs causing MG. It is detected among approximately $80 \%$ of patients with MG (1). In 2001, Hoch et al. reported that $70 \%$ of AChR-Abs-negative patients with MG possess IgGs with the capacity to bind with muscle-specific kinase (MuSK) (2). MuSK MG predominantly occurs in women with frequent oculobulbar symptoms and such patients are at higher risk of developing MG crises. Anti-low-density lipoprotein receptor-antigen (Lrp4) Abs is a recently discovered novel autoantibody, and its positivity among patients with MG is reported to be $2-50 \%$ (3-7). Although a previous study reported the rare coexistence of MuSK and Lrp4 Abs among AChR-Ab-negative patients with MG, its detailed clinical characteristics remain to be elucidated. Antistriational antibodies such as anti-titin, anti-ryanodine receptor (RyR), and muscular voltage-gated potassium channelcomplex (Kv1.4) are also known to be MG-associated anti- bodies. Anti-titin Abs most frequently coexist in anti-AChR Abs-positive MG and they have been shown to be associated with myositis or cardiomyopathy (8). We herein report the clinical presentations, laboratory characteristics, and therapeutic response of a patient with MG who was positive for anti-MuSK, anti-Lrp4 and anti-titin Abs.

\section{Case Report}

A 62-year-old woman had been suffering from intermittent double vision, ptosis, and dropped head since her fifties without undergoing any regular medical examination. One morning, she noticed unprecedented fatigue and respiratory discomfort. In the evening, she became unconscious and was brought in for emergency care. When she arrived at our hospital, she was completely unconscious (Glasgow Coma Scale 1-1-4). Her respiration was also in a state of arrest, and pulse arterial oxygen saturation was not detectable. Her blood pressure was 105/83 $\mathrm{mmHg}$. An arterial blood gas test showed respiratory acidosis $\left(\mathrm{pH} 7.054, \mathrm{PCO}_{2} 138 \mathrm{mmHg}\right.$, $\mathrm{PO}_{2} 123 \mathrm{mmHg}, \mathrm{HCO}_{3} 36.7 \mathrm{mEq} / \mathrm{L}, \mathrm{BE} 0.8 \mathrm{mmol} / \mathrm{L}$, and lactate concentration $41 \mathrm{mEq} / \mathrm{L})$. An electrocardiogram showed sinus tachycardia. Brain magnetic resonance imag-

${ }^{1}$ Department of Neurology, Osaka University Hospital, Japan and ${ }^{2}$ Department of Clinical Research, Nagasaki Kawatana Medical Center, Japan Received: April 3, 2020; Accepted: July 1, 2020; Advance Publication by J-STAGE: August 22, 2020

Correspondence to Dr. Tatsusada Okuno, okuno@neurol.med.osaka-u.ac.jp 

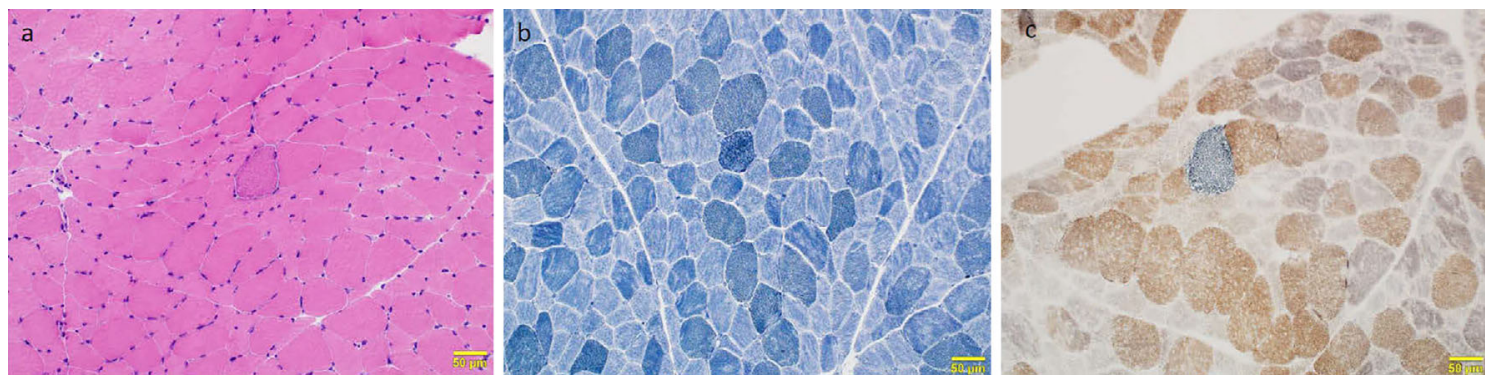

Figure 1. Muscle pathology. A muscle biopsy from the biceps brachii. a) On Hematoxylin and Eosin staining, mild to moderate fiber size variation without inflammatory cell infiltration is visible. b) On nicotinamide adenine dinucleotide tetrazolium reductase (NADH-TR) staining, disorganized intermyofibrillar network is observed in some fibers. c) On COX \& SDH staining, a COX negative fiber is visible.

ing and chest and abdominal contrast computed tomography (CT) images were normal. Within a few hours after she was placed under artificial ventilation, her consciousness gradually recovered to a normal state. A neurological examination revealed facial and neck flexion weakness. She was not able to close her eyes completely, she also had puffy cheeks, but she could raise her head up from the bed [manual muscle test (MMT) 2/5]. Her ocular movement was limited, especially in the horizontal direction. Bilateral ptosis was also observed. After being extubated, she also showed severe dysphagia. No signs of limb muscle weakness were observed. Her deep tendon reflexes were preserved without any signs of pyramidal involvement. Nerve conduction studies of the median, ulnar, tibial, and sural nerves showed normal results. Repetitive nerve stimulation of the abductor digiti minimi and trapezius muscles was also normal. An electromyogram (EMG) showed early recruitment. Neither fibrillation nor fasciculation potentials were detected. Her cardiac function was also normal. Therefore, a muscle biopsy was performed and revealed myopathic changes. On Hematoxylin and Eosin (H\&E) staining, mild to moderate fiber size variation was observed without inflammatory cell infiltration. Atrophied fibers were mainly type 2 . Slight fibers with internal nuclei and cytochrome oxidase (COX) negative fibers were observed, and the intermyofibrillar network was disorganized (Fig. 1). Anti-MuSK Ab measured by a radioimmunoassay (RIA) was $28.6 \mathrm{nmol} / \mathrm{L}$ (normal, <0.02). AntiLrp4 Ab measured by luciferase immunoprecipitation systems was 62,568 relative light units (RLU) (positive control, 58,682 RLU). Anti-titin Ab measured by cytometric cell based assay was 1.18 (normal range <1.0). Anti-AChR evaluated by RIA was negative. anti-Kv1.4 Ab, and myositis-associated Abs, such as anti-mitochondria, antiaminoacyl tRNA synthetase (ARS), anti-Mi-2, anti-3hydroxy-3-methylglutaryl-coenzyme A reductase (HMGCR), and signal recognition particle antibody, were negative. Based on the clinical presentation and positivity of antiMuSK Abs, the patient was diagnosed with MuSK MG with anti-Lrp4 and anti-titin Abs [Myasthenia Gravis Foundation of America (MGFA) Class V]. Her serum creatine kinase level was not elevated. Normal chest CT findings indicated the absence of thymoma. Two weeks after the admission, she was able to maintain her respiratory function without ventilator support during the daytime, although her forced lung capacity (FLC) was 53.8\%. Her neck flexor strength also persisted (MMT 2), and she was still unable to even swallow even saliva. High-dose intravenous methylprednisolone (1,000 mg daily for 3 days) and oral tacrolimus (3 mg/ day) were initiated at 1 month after admission. Her respiratory function gradually recovered after the first steroid pulse therapy, and ventilator support was thereafter completely removed. Additional high-dose steroid therapy was administered twice at 10-day intervals, followed by $10 \mathrm{mg} /$ day of oral prednisolone. After the last steroid pulse therapy, her neck flexion strength gradually recovered (MMT 4), and her FLC recovered to $95.3 \%$, although dysphagia remained. Maintenance therapy was then changed from tacrolimus to cyclosporine $(5 \mathrm{mg} / \mathrm{kg}$ ), in addition to pyridostigmine. After 60 days of admission, she gradually became able to swallow soft foods and was discharged from the hospital 72 days after admission (Fig. 2).

\section{Discussion}

We herein describe the case of a MuSK MG patient accompanied by anti-Lrp4 and anti-titin Abs. To the best of our knowledge, this is the first detailed report of a case with triple antibody positive MuSK MG. MuSK and Lrp4 are related to agrin/Lrp4/MuSK signaling. Neural agrin induces AChR clustering necessary for neuromuscular junction (NMJ) formation. Agrin binding to Lrp4 and MuSK triggers this signaling pathway $(9,10)$. Agrin binding to Lrp4 has been reported to form a heterodimer, and two of them generate a tetramer that initiates MuSK activation. Lrp4 and MuSK can interact with each other without agrin; however, the interaction is increased by agrin $(9,10)$. The clinical features of anti-MuSK Abs-positive MG are characterized by a female predominance with frequent oculobulbar weakness and high risk of MG crises (11). Jeffery et al. reviewed 110 patients with anti-MuSK Abs-positive MG, and 85\% of them were female. Ocular or bulbar symptoms at the onset were present in $79 \%$ of these patients. Approximately $85 \%$ 


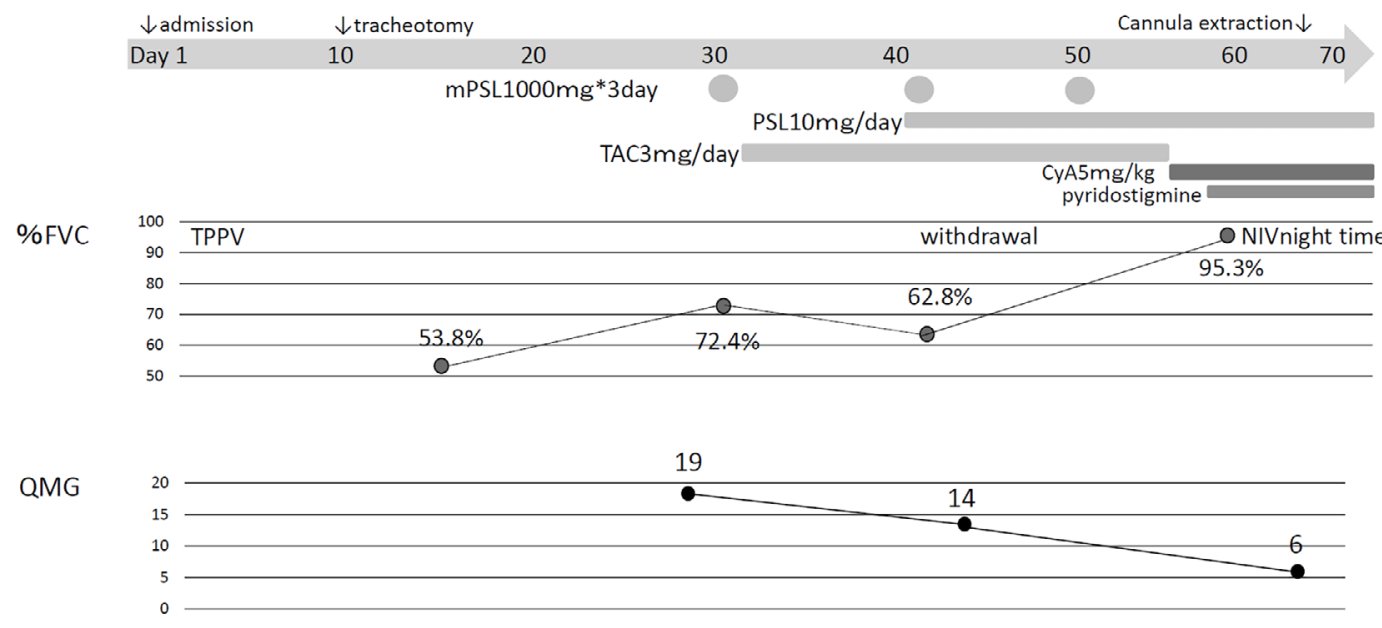

Figure 2. Clinical course. mPSL: methylprednisolone, PSL: prednisolone, TAC: tacrolimus, CyA: Cyclosporine A, TPPV: tracheostomy positive pressure ventilation, NIV: non-invasive ventilation, QMG: quantitive myaethenia gravis score, FVC: forced vital capacity

of patients were classified to have more severe disease than MGFA Class III. MG crises occurred in $28 \%$ among the patient cohort. Lrp4 was initially reported to be a novel target antigen in seronegative $\mathrm{MG}$, while accumulating evidence suggests that anti-Lrp4 Abs also becomes positive in other neuromuscular diseases. For example, in amyotrophic lateral sclerosis patients, anti-Lrp4 Ab positivity is reported to be $23.4 \%$ which is apparently a higher rate than that of $\mathrm{MG}$ patients (12). In this regard, the pathogenic significance of anti-Lrp4 $\mathrm{Ab}$ remains to be elucidated. According to Zisimopoulou et al. (6), patients with MG with anti-Lrp4 Abs tend to present with either ocular or mild generalized symptoms. Although bulbar symptoms are high $(66 \%)$, $85 \%$ of patients with anti-Lrp4 Abs were classified as MGFA Class I or II, and none belonged to MGFA Class V, whereas only $51 \%$ and $52 \%$ of anti-AChR and anti-MuSK Abs-positive patients with MG were classified as MGFA Class I or II, respectively. These observations suggest that patients with Lrp4-MG have milder symptoms. Patients with Lrp4-MG are commonly treated with pyridostigmine and corticosteroids (6). Anti- Lrp4-Abs coexists with either anti-AChR or anti-MuSK Abs at various degrees according to previous studies. According to Zisimopoulou et al., 7.5\% of AChRMG patients and $14.6 \%$ MuSK MG patients possess coexisting serum anti- Lrp4 Abs. Seven out of 8 patients with AChR/Lrp4 MG and all 8 patients with MuSK/Lrp4 MG presented with MGFA Class III or IV. MG crises were observed in $12.5 \%$ of patients with $\mathrm{AChR} / \mathrm{Lrp} 4 \mathrm{MG}$ and in $62.5 \%$ with MuSK/Lrp4. None of the patients with MuSK/ Lrp4 MG were accompanied by thymoma or thymic hyperplasia. All of patients with MuSK/Lrp4 MG showed a good or moderate response to pyridostigmine and prednisone treatment, whereas patients with AChR/Lrp4 MG showed a much better response to pyridostigmine. Taken together, MuSK/Lrp4 MG showed relatively severe clinical symptoms, a frequent rate of $\mathrm{MG}$ crises, and a good response to pyridostigmine and prednisone, in comparison to AChR/
Lrp4 MG (6). Consistent with the previous cohort study, our patient showed MG crisis with severe bulbar symptoms, which effectively responded to high-dose steroid and oral immunosuppressive therapy. In our case, the patient had a 10-year history of MG symptoms. Her symptoms were mild at onset, and then gradually became aggravated. Whether she possessed all Abs from the onset or only one or two Abs at the beginning could not be clarified in our case. It is noteworthy that anti-titin $\mathrm{Ab}$ was also found in this case. Anti-titin Abs is one of the anti-striational Abs that mainly appears in anti-AChR Abs-positive MG (8) and has been shown to be associated with myositis or cardiomyopathy. Consistently, EMG and a muscle biopsy revealed myopathic changes in this case, but no infiltration centered on CD8positive $\mathrm{T}$ cell was found, unlike that of previous studies (13). Although $\mathrm{T}$ cell infiltration was not evident in our case, anti-titin Ab might have contributed to augment disease severity of MG by accelerating autoimmune reaction not only against neuromuscular junction, but against muscle fibers. It is reported that $40.9 \%$ of AChR-MG, $14.6 \%$ of MuSK-MG and $16.4 \%$ of Lrp4-MG patients possess antititin $\mathrm{Ab}$ (14). Thus, our case further supports the previous observation that anti-titin Abs occasionally coexist with antiAChR negative MG.

In conclusion, the clinical features of double- or triplepositive patients would help us reveal the clinical spectrum of the disease. Notably, this case showed that not only neuromuscular junctions, but also muscle cell bodies may have been targeted for autoimmune responses in anti-MuSKAb positive MG accompanied by anti-Lrp4 and anti-titin antibodies.

The authors state that they have no Conflict of Interest (COI).

\section{Acknowledgement}

We express our sincere thanks to Dr. Ichizo Nishino at the National Center of Neurology and Psychiatry for the diagnosis of 
the muscle biopsy findings.

\section{References}

1. Leite MI, Waters P, Vincent A. Diagnosic use of autoantibodies in myasthenia gravis. Autoimmunity 43: 371-379, 2010.

2. Hoch W, Mcconville J, Helms S, Newsom-Davis J, Melms A, Vincent A. Auto-antibodies to the receptor tyrosine kinase MuSK in patients with myasthenia gravis without acetylcholine receptor antibodies. Nat Med 7: 365-368, 2001.

3. Higuchi O, Hamuro J, Motomura M, Yamanashi Y. Autoantibodies to low-density lipoprotein receptor-related protein 4 in myasthenia gravis. Ann Neurol 69: 418-422, 2011.

4. Pevzner A, Schoser B, Peters K, et al. Anti-LRP4 autoantibodies in AChR- and MuSK-antibodynegative myasthenia gravis. J Neurol 259: 427-435, 2012.

5. Zhang B, Tzartos JS, Belimezi M, et al. Autoantibodies to lipoprotein-related protein 4 in patients with double-seronegative myasthenia gravis. Arch Neurol 69: 445-451, 2012.

6. Zisimopoulou P, Evangelakou P, Tzartos J, et al. A comprehensive analysis of the epidemiology and clinical characteristics of antiLRP4 in myasthenia gravis. J Autoimmun 52: 139-145, 2014.

7. Cossins J, Belaya K, Zoltowska K, et al. The search for new antigenic targets in myasthenia gravis. Ann N Y Acad Sci 1275: 123 128, 2012.
8. Suzuki S, Utsugisawa K, Nagane Y, Suzuki N. Three types of striational antibodies in myasthenia gravis. Autoimmune Dis. Forthcoming.

9. Kim N, Stiegler AL, Cameron TO, et al. Lrp4 is a receptor for agrin and forms a complex with MuSK. Cell 135: 334-342, 2008.

10. Zhang B, Luo S, Wang Q, Suzuki T, Xiong WC, Mei L. LRP4 serves as a coreceptor of agrin. Neuron 60: 285-297, 2008.

11. Guptill JT, Sanders DB, Evoli A. Anti-MuSK antibody myasthenia gravis: clinical findings and response to treatment in two large cohorts. Muscle Nerve 44: 36-40, 2011.

12. Tzartos JS, Zisimopoulou P, Rentzos, et al. LRP4 antibodies in serum and CSF from amyotrophic lateral sclerosis patients. Ann Clin Transl Neurol 1: 80-88, 2014.

13. Suzuki S, Utsugisawa K, Yoshikawa $\mathrm{H}$, et al. Autoimmune targets of heart and skeletal muscles in myasthenia gravis. Arch Neurol 66: 1334-1338, 2009.

14. Stergiou C, Lazaridis K, Zouvelou V. Titin antibodies in "seronegative" myasthenia gravis-a new role for an old antigen. J Neuroimmunol 292: 108-115, 2016.

The Internal Medicine is an Open Access journal distributed under the Creative Commons Attribution-NonCommercial-NoDerivatives 4.0 International License. To view the details of this license, please visit (https://creativecommons.org/licenses/ by-nc-nd/4.0/).

(C) 2021 The Japanese Society of Internal Medicine Intern Med 60: 137-140, 2021 